\title{
Produção de frutos e incidência de cancro cítrico em laranjeiras "Monte Parnaso" enxertadas sobre sete porta-enxertos
}

\author{
Fruit production and incidence of citrus canker lesions on "Monte Parnaso" navel \\ orange budded on seven rootstocks
}

\author{
Bernadete Reis ${ }^{\mathrm{I}}$ Otto Carlos Koller ${ }^{\mathrm{II}}$ Sergio Francisco Schwarz ${ }^{\mathrm{II}}$ Sergiomar Theisen ${ }^{\mathrm{I}}$ \\ Ivar Antônio Sartori ${ }^{\mathrm{I}}$ Fernanda Severo Nichele ${ }^{\mathrm{III}}$ Rafael Lorscheiter ${ }^{\mathrm{III}}$ \\ Henrique Belmonte Petry ${ }^{\text {III }}$
}

\section{RESUMO}

Porta-enxertos de citros podem exercer influência sobre a produção de frutos e suscetibilidade das plantas ao cancro cítrico. Este trabalho objetivou selecionar porta-enxertos que induzam elevado índice de produtividade e menor suscetibilidade das copas de laranjeiras-de-umbigo "Monte Parnaso"(Citrus sinensis) à bactéria Xanthomonas axonopodis pv. citri, causadora do cancro cítrico (CC). O experimento foi desenvolvido em um pomar experimental com 10 anos de idade, plantado em espaçamento de $2,5 \times 6,0 m$, no município de Butiá, no Estado do Rio Grande do Sul. O delineamento experimental foi em blocos ao acaso, com sete tratamentos e quatro repetições, usando duas plantas por parcela. Os tratamentos constaram dos seguintes porta-enxertos: limoeiros "Cravo" (C. limonia) e "Volkameriano" (C. volkameriana.), trifoliata "Flying Dragon" (Poncirus trifoliata.), laranjeira "Caipira" (C. sinensis), citrangeiro "Troyer" (C. sinensis x $\boldsymbol{P}$. trifoliata), tangerineira "Sunki" (C. sunki.) e citrumeleiro "Swingle" (C. paradisi x P. trifoliata). Os parâmetros avaliados foram: incidência de CC em folhas e frutos em toda a planta e em ramos previamente selecionados, em diversas épocas. Verificou-se que as plantas enxertadas sobre citrumeleiro "Swingle" apresentaram o maior índice de produtividade e, à semelhança do "Flying Dragon", a menor incidência de CC, tanto nas folhas como nos frutos. Além disso, verificou-se também que as plantas enxertadas sobre os limoeiros "Cravo" e "Volkameriana", apesar de produzirem as mais elevadas cargas de frutos, foram as mais suscetíveis ao CC.

Palavras chave: Citrus sinensis, Xanthomonas axonopodis pv. citri resistência genética.

\section{ABSTRACT}

Citrus rootstocks can exert some influences on fruit production and susceptibility of the plants to citrus canker (CC). This research was performed aiming to select rootstocks which induce higher productivity index and lower susceptibility of 'Monte Parnaso' navel orange (Citrus sinensis) trees to the bacteria Xanthomonas axonopodis pv. citri. The research was carried out in an experimental orchard, with ten-years-old trees, planted at spacement of $2.5 \mathrm{~m}$ between trees and $6.0 \mathrm{~m}$ between rows, located in Butiá city, in the Central Depression of Rio Grande do Sul state. It was used an randomized blocks experimental design, with 7 treatments and 4 replications. The treatments consisted of the following rootstoks: Rangpur lime (C. limonia.), 'Volkameriana' lemon (C. volkameriana.), 'Flying Dragon' trifoliata orange (Poncirus trifoliata), 'Caipira' orange (C. sinensis), 'troyer' citrange (C. sinensis $x$ P. trifoliata), 'Sunki' tangerine (C. sunki.) and 'Swingle' citrumelo (C. paradisi $x$ P. trifoliata). The parameters evaluated were: number of fruits and leaves with citrus canker lesions per plant and at two previously selected and marked branches per plant, at different times. The results revealed that the 'Swingle' citrumelo and 'Flying dragon' rootstocks induced the highest productivity index and, the lowest incidence of citrus canker disease on leaves and fruits. 'Rangpur' lime and 'Volkameriana' lime rootstocks, promoted a heavy crop load, however, showed higher susceptibility to citrus canker disease.

Key words: Citrus sinensis, Xanthomonas axonopodis pv. citri genetic resistance.

\section{INTRODUÇÃO}

O cancro cítrico, causado pela bactéria Xanthomonas axonopodis pv. citri (Hasse, 1915, Auterin et al., 1995), é uma doença quarentenária A2 (RODRIGUES NETO \& RIBEIRO, 2002), considerada uma das principais doenças da cultura dos citros, por ocasionar queda de folhas e lesões em frutos,

\footnotetext{
'Programa de Pós-graduação em Fitotecnia, Universidade Federal do Rio Grande do Sul (UFRGS), Porto Alegre, RS, Brasil.

IDepartamento de Horticultura e Silvicultura, Faculdade de Agronomia, UFRGS. Av. Bento Gonçalves, 7712, 91591-970, Porto

Alegre, RS, Brasil. E-mail: ockoller@adufrgs.br. *Autor para correspondência.

${ }^{\text {IIIC } C u r s o ~ d e ~ A g r o n o m i a, ~ U F R G S, ~ P o r t o ~ A l e g r e, ~ R S, ~ B r a s i l . ~}$
} 
prejudicando a produção, a qualidade visual, a comercialização e exportação de frutos (CHRISTIANO, 2003; OLIVEIRA et al., 2001). A doença manifesta-se em todos os órgãos da parte aérea das plantas cítricas. Seus sintomas são bastante característicos, variando conforme a espécie, a cultivar, a idade e os órgãos afetados (LEITE JUNIOR, 1990), sendo que as cultivares de citros apresentam diversos graus de resistência à Xanthomonas axonopodis pv. citri, segundo observações de NAMEKATA et al. (1992), LEITE JUNIOR (1990) e ROSSETTI (2001). No entanto, existe pouca informação a respeito da eventual influência do porta-enxerto sobre a severidade da doença na variedade-copa (LEITE JUNIOR \& SANTOS, 1988).

O porta-enxerto exerce grande influência em diversas características da variedade-copa (WUSTCHER, 1979), podendo influir também na suscetibilidade ao cancro cítrico. Na província de Missiones, Argentina, AGOSTINI et al. (1985) correlacionaram positivamente a incidência e a severidade da doença com o maior vigor induzido. Tendo em vista que somente frutos imaturos, folhas e ramos novos são suscetíveis ao CC, a maior severidade da doença em plantas enxertadas sobre porta-enxertos vigorosos pode ser atribuída à maior freqüência e duração dos períodos de brotação, promovida pelos porta-enxertos vigorosos (DANOS et al., 1981; 1984). Assim, LEITE JUNIOR \& SANTOS (1988) verificaram que a incidência da doença foi maior em plantas enxertadas sobre Citrus karna Raf. e sobre limoeiros "Rugoso-da-África” e "Cravo", sendo menor em plantas enxertadas sobre o "Trifoliata" [Poncirus trifoliata (L.) Raf.] (ROSSETTI , 2001).

Buscou-se, neste trabalho, verificar a influência de diversos porta-enxertos sobre a produção de frutos, bem como estudar o comportamento do cancro cítrico e sua incidência em laranjeiras-de-umbigo "Monte Parnaso", em diversas épocas, com o objetivo de selecionar porta-enxertos que possam ser recomendados como alternativa ao "Trifoliata". Este porta-enxerto é de uso dominante no Rio Grande do Sul, o que pode representar sérios riscos aos pomares se surgirem outras doenças às quais o "Trifoliata" possa tornar-se suscetível no futuro.

\section{MATERIAL E MÉTODOS}

O experimento foi realizado com laranjeirasde-umbigo "Monte Parnaso” [Citrus sinensis (L.) Osb.] com 10 anos de idade, enxertadas sobre sete portaenxertos, num pomar experimental com espaçamento de plantio de 2,5m entre plantas e $6 \mathrm{~m}$ entrelinhas, situado no município de Butiá, Estado do Rio Grande do Sul, em solo Podzólico Vermelho-Escuro, de textura francoargilosa. O clima é do tipo Cfa, subtropical sem estação seca definida, segundo a classificação de Köeppen.

O delineamento foi em blocos ao acaso, com sete tratamentos e quatro repetições. Os blocos foram dispostos em linhas paralelas de laranjeiras, sem utilização de plantas bordadura entrelinhas. Cada parcela foi constituída de quatro plantas na linha, das quais apenas as duas centrais foram avaliadas. Os porta-enxertos testados foram: limoeiros "Cravo" (Citrus limonia Osb.) e "Volkameriana” (Citrus volkameriana Pas.), “Flying Dragon” (P. trifoliata), laranjeira "Caipira” (Citrus sinensis), citrangeiro “Troyer” (C. sinensis x P. trifoliata), tangerineira “Sunki” (C. sunki Hort. ex Tan.) e citrumeleiro "Swingle" (C. paradisi x P. trifoliata).

O pomar experimental foi instalado em julho de 1996. As práticas culturais, compreendendo adubações químicas e controle de pragas, de doenças e da vegetação espontânea, foram sempre as mesmas em todos os tratamentos. Em 1999, foi constatado que o pomar havia sido infectado pelo CC. Na tentativa de erradicação da doença, sem sucesso, durante dois anos consecutivos, as plantas com sintomas foram severamente podadas e pulverizadas a cada 21 dias com oxicloreto de cobre. A partir de 2002, a poda sanitária foi suspensa e passou-se então a controlar o CC somente com cinco pulverizações anuais de oxicloreto de cobre, na concentração de $0,15 \%$ de cobre metálico, três delas feitas nos principais surtos de brotação (setembro, novembro/dezembro e fevereiro) e outras duas em outubro e em janeiro.

Os parâmetros avaliados, seguindo metodologias testadas por THEISEN (2004) e MONZANI (2005), foram: número e porcentagem de folhas com CC, número e massa de frutos que apresentavam pelo menos uma lesão de CC e número e massa total dos frutos produzidos por planta. Em março e maio de 2004, a avaliação da incidência de CC constou da retirada e contagem de todas as folhas da planta com sintomas da doença. Em dezembro de 2004 e em janeiro, março e abril de 2005, foram efetuadas avaliações em dois ramos previamente marcados, com fita plástica branca. Em cada planta útil, nos dois lados da linha de plantio (norte e sul), na altura média da copa, contou-se o número de folhas com e sem lesões de CC, porém sem retirá-las dos ramos. Já na última avaliação, em junho de 2005, foram novamente retiradas todas as folhas com lesões. A colheita dos frutos foi realizada em setembro de 2004 e em setembro de 2005, sendo contados e pesados, em separado, os frutos com e sem lesões de CC. 
O crescimento das plantas foi avaliado medindo-se o diâmetro das copas nos sentidos longitudinal (DL) e transversal (DT) à linha de plantio. De posse desses dados determinou-se o espaço ocupado pelas plantas: área da projeção da copa (APC), aplicando-se a fórmula: APC $=$ p. $(\mathrm{DL}+\mathrm{DT} / 4)^{2}$, utilizada por SCHAFER et al. (2001) e MARTINS (2005). O índice de produtividade (IP), em cada porta-enxerto, foi determinado pela divisão da massa total de frutos produzidos pela APC.

Os dados relativos ao número e à porcentagem de folhas e frutos com lesões de CC foram analisados em esquema fatorial, sendo os fatores utilizados as épocas de avaliação e os porta-enxertos. Para a análise da variância, os dados foram transformados em $(\mathrm{x}+1)^{1 / 2}$ e as diferenças entre médias de tratamentos foram verificadas pela aplicação do teste de Duncan a 5\% de probabilidade de erro.

\section{RESULTADOS E DISCUSSÃO}

Em março e em maio de 2004 (Tabela 1), em toda a copa, nas duas avaliações, as plantas enxertadas sobre o limoeiro “Cravo” apresentaram maior número de folhas com lesões de CC (NFoCC), seguidas pelas da tangerineira "Sunki”, do limoeiro "Volkameriano" e do citrangeiro "Troyer”, que não diferiram entre si. O porta-enxerto “Flying Dragon” apresentou o menor número de folhas com lesões de CC.

Pode-se verificar que, na avaliação feita em maio, o NFoCC foi bem menor do que na março. Esta redução se justifica porque, em princípio, nesta avaliação, foram incluídas somente as folhas infectadas a partir do mês de março, enquanto que na avaliação anterior foram computadas folhas que estavam sujeitas a serem infectadas durante um período muito maior, envolvendo inclusive o ciclo vegetativo do ano anterior. Entretanto, essa redução também pode ter sido favorecida pela diminuição da temperatura, que, de março a maio, ficou entre $17^{\circ} \mathrm{C}$ e $23^{\circ} \mathrm{C}$, diminuindo a brotação das plantas e a colonização do CC.

Nas avaliações da \%FoCC, realizadas em ramos previamente marcados, de dezembro de 2004 a junho de 2005 (Tabela 2), não ocorreu interação significativa entre épocas e porta-enxertos. Isso indica que, em todas as épocas, os porta-enxertos evidenciaram o mesmo comportamento com relação à incidência de CC nas folhas, sendo que, na soma dos valores das diversas épocas de avaliação, as maiores \%FoCC ocorreram nas plantas enxertadas sobre os limoeiros "Cravo", "Volkameriano" e sobre a tangerineira "Sunki”, ao passo que as plantas enxertadas sobre "Flying Dragon" e citrumeleiro "Swingle" foram as menos infectadas. Esse comportamento dos porta-enxertos, com relação à incidência do CC, revelou-se similar ao já detectado pelo método da contagem das folhas lesionadas de toda a copa.

Nas avaliações de março e abril de 2005 (Tabela 2), houve redução do NFoCC, em relação a dezembro de 2004 e janeiro de 2005, em todos os portaenxertos, o que pode ser atribuído à abscisão natural de folhas lesionadas. Porém, segundo SCHUBERT \& SUN (2003), as lesões de CC, dependendo de condições climáticas favoráveis ou desfavoráveis, principalmente da temperatura ambiente, tornam-se visíveis, a olho nu, somente dos quatro aos 60 dias após a penetração das bactérias no tecido. Assim sendo, os índices de incidência apurados em cada época de avaliação são o reflexo de infecções que ocorreram com antecedência de 4 ou mais dias. Então, o elevado NFoCC que se

Tabela 1 - Número de folhas com lesões de cancro cítrico (NFoCC) em toda a copa de laranjeiras-de-umbigo "Monte Parnaso” enxertadas sobre sete porta-enxertos. Março e maio de 2004. Butiá, RS, 2005.

\begin{tabular}{|c|c|c|c|}
\hline \multirow{2}{*}{ Porta-enxertos } & \multicolumn{2}{|c|}{ Toda copa 2004} & \multirow{2}{*}{ Soma FoCC março/maio } \\
\hline & março & maio & \\
\hline \multicolumn{4}{|c|}{ Folhas com lesões de CC } \\
\hline Citrumeleiro "Swingle" & A $717,8 \mathrm{bc}$ & B $163,5 b$ & 881,3 bc \\
\hline Tangerineira “Sunki” & A 962,0ab & B 217,0ab & $1179,0 \mathrm{ab}$ \\
\hline Limoeiro "Volkameriana” & A 938,3ab & B 294,8ab & $1233,1 \mathrm{ab}$ \\
\hline Limoeiro “Cravo” & A $1106,5 a$ & В 446,3а & 1552,8 a \\
\hline “Flying Dragon” & A $217,0 \mathrm{~d}$ & B 10,0 c & $227,0 \mathrm{~d}$ \\
\hline Citrangeiro “Troyer” & A 856,5ab & B 355,5 ab & $1212,0 \mathrm{ab}$ \\
\hline Laranjeira “Caipira” & A $469,0 c$ & B 165,5 b & 634,5 c \\
\hline $\mathrm{CV}$ & & 20,28 & \\
\hline
\end{tabular}

Médias antecedidas de mesma letra maiúscula na linha e seguidas de letra minúscula na coluna não diferem significativamente pelo teste de Duncan a $5 \%$ de probabilidade.

Ciência Rural, v.38, n.3, mai-jun, 2008. 
Tabela 2 - Porcentagem de folhas com lesões de cancro cítrico em ramos marcados de laranjeiras-de-umbigo "Monte Parnaso” enxertadas sobre sete porta-enxertos. Dezembro de 2004 a junho de 2005. Butiá, RS. 2005.

\begin{tabular}{|c|c|c|c|c|c|c|}
\hline Porta-enxertos & dez 2004 & jan & mar & abril & junho/2005 & Soma \\
\hline & \multicolumn{6}{|c|}{$\%$ FoCC } \\
\hline Citrumeleiro "Swingle" & 1,78 & 1,49 & 0,71 & 0,69 & 0,76 & $5,43 \mathrm{~cd}$ \\
\hline Tangerineira "Sunki” & 2,20 & 3,46 & 1,57 & 1,16 & 3,29 & $11,68 \mathrm{ab}$ \\
\hline Limoeiro “Volkameriana” & 2,26 & 3,34 & 1,47 & 1,25 & 4,22 & $12,54 \mathrm{a}$ \\
\hline Limoeiro “Cravo” & 2,51 & 3,43 & 2,06 & 1,33 & 4,10 & $13,43 \mathrm{a}$ \\
\hline “Flying Dragon” & 0,88 & 0,87 & 0,47 & 0,15 & 0,68 & $3,05 \mathrm{~d}$ \\
\hline Citrangeiro “Troyer" & 1,75 & 2,78 & 1,65 & 0,63 & 1,99 & 8,80 bc \\
\hline Laranjeira “Caipira” & 1,52 & 1,96 & 1,42 & 1,29 & 2,54 & $9,73 \mathrm{bc}$ \\
\hline Média época & $1,84 \mathrm{~A}$ & $2,47 \mathrm{~A}$ & 1,33 B & 0,92 B & $2,51 \mathrm{~A}$ & \\
\hline $\mathrm{CV}$ & \multicolumn{6}{|c|}{34,39} \\
\hline
\end{tabular}

Médias seguidas de mesma letra maiúscula na linha e minúscula na coluna não diferem significativamente pelo teste de Duncan a 5\% de probabilidade.

verificou na avaliação de janeiro de 2005 foi decorrente da existência de condições de ambiente favoráveis à doença em dezembro de 2004. Porém, as baixas precipitações pluviométricas de 33mm em janeiro e 64mm em fevereiro de 2005 foram desfavoráveis à colonização do CC neste período, diminuindo o NFoCC presente na avaliação de abril. Além disso, as carências hídricas também diminuem a brotação das plantas, reduzindo a presença de tecidos suscetíveis ao CC (CANTEROS, 2000).

Na avaliação feita em junho (Tabela 2), com a retirada e contagem de todas as folhas que apresentavam lesões, o NFoCC aumentou. Este resultado foi surpreendente, porque no outono/ inverno, normalmente, devido às baixas temperaturas, escasseiam as brotações das plantas e as bactérias se tornam menos ativas. Conseqüentemente, de abril a junho, não deveriam ter surgido novas lesões, tanto assim que, na Argentina, é recomendável fazerem-se quatro a cinco pulverizações cúpricas protetivas para controlar o CC (CANTEROS, 2000). No experimento, pode ter acontecido que, na leitura feita em abril, algumas folhas de ramos novos já tivessem sido infectadas pelas bactérias, sem terem ainda evidenciado os sintomas. Portanto, essas lesões se tornariam visíveis só algum tempo mais tarde e, como no intervalo não foram feitas verificações, as lesões só foram computadas em junho. Observou-se também que, no período de abril/maio, houve temperaturas em torno de $25^{\circ} \mathrm{C}$ e chuvas de até $190 \mathrm{~mm}$, suficientemente altas para possibilitarem a brotação das plantas e as infecções de CC (AMARAL, 2003; VERNIERE et al., 2003).

A constatação desse aumento da incidência de CC, no período de abril a junho, sugere que, para a cultivar "Monte Parnaso", que é muito suscetível a essa doença, dependendo da região de cultivo e das condições climáticas de cada ano, além das cinco pulverizações cúpricas recomendadas para laranjeiras na Argentina, poderia ser conveniente a realização de uma sexta aplicação, em abril, para reduzir a ocorrência de infecções nesta época. Assim, seria possível diminuir a presença de lesões, que são as principais fontes de inóculos na re-infecção dos tecidos jovens que surgem na brotação primaveril, em setembro.

A maior incidência de cancro cítrico esteve relacionada à maior área de projeção da copa (APC), motivada pelo porta-enxerto (Tabela 3). Isso coincide, em parte, com pesquisas realizadas por LEITE JUNIOR \& SANTOS (1988) sobre a maior suscetibilidade das plantas enxertadas sobre os porta-enxertos mais vigorosos, e com dados de DANOS et al. (1981; 1984) e AGOSTINI et al. (1985), que verificaram a existência de correlação positiva entre os porta-enxertos vigorosos e a maior incidência de CC, sendo que esses os surtos de brotação são mais freqüentes e prolongados (AGOSTINI et al., 1985; WUTSCHER, 1979).

As copas de limoeiros “Siciliano” enxertadas sobre o $\boldsymbol{P}$. trifoliata apresentaram níveis mais baixos de cancro cítrico, entre diversos porta-enxertos testados (LEITE JUNIOR \& SANTOS, 1988). O $\boldsymbol{P}$. trifoliata é um dos porta-enxertos que induzem menor vigor às plantas cítricas, o que pode explicar a menor incidência da doença no "Flying Dragon” observada nessa pesquisa, uma vez que esse porta-enxerto é um mutante do $\boldsymbol{P}$. trifoliata, que reduz ainda mais o vigor das plantas sobre ele enxertadas.

A semelhança de respostas, entretanto, indica que, nos porta-enxertos mais vigorosos, a maior incidência de cancro cítrico não se deveu simplesmente ao maior volume de copa e número de folhas das 
Tabela 3 - Área de projeção da copa (APC), soma de folhas com lesões de cancro cítrico (FoCC) em toda a copa e porcentagem de folhas com lesões (\%FoCC) em ramos de laranjeiras-de-umbigo "Monte Parnaso" enxertadas sobre sete porta-enxertos 2004 e 2005. Butiá, RS. 2005.

\begin{tabular}{|c|c|c|c|c|}
\hline Porta-enxertos & 2004 & 2005 & Сopa/2004 & Ramo/2005 \\
\hline & \multicolumn{2}{|c|}{$\operatorname{APC}\left(\mathrm{m}^{2}\right)$} & FoCC & \%FoCC \\
\hline Citrumeleiro "Swingle" & 3,31 a & $3,34 \mathrm{a}$ & 881,3 bc & $5,43 \mathrm{~cd}$ \\
\hline Tangerineira “Sunki” & $3,18 \mathrm{a}$ & $3,36 \mathrm{a}$ & $1179,0 a b$ & $11,68 \mathrm{ab}$ \\
\hline Limoeiro "Volkameriana” & 3,45 a & 3,89 a & $1233,1 \mathrm{ab}$ & 12,54 a \\
\hline Limoeiro “Cravo” & $4,01 \mathrm{a}$ & $4,70 \mathrm{a}$ & 1552,8 a & 13,43 a \\
\hline “Flying Dragon” & $1,20 \mathrm{~b}$ & $1,22 \mathrm{~b}$ & $227,0 \mathrm{~d}$ & $3,05 d$ \\
\hline Citrangeiro “Troyer” & 3,43 a & 3,79 a & $1212,0 \mathrm{ab}$ & 8,80 bc \\
\hline Laranjeira “Caipira” & $3,31 \mathrm{a}$ & $3,42 \mathrm{a}$ & 634,5 c & 9,73 bc \\
\hline $\mathrm{CV}$ & 7,34 & 6,50 & 20,58 & 34,39 \\
\hline
\end{tabular}

Médias seguidas de mesma letra na coluna não diferem significativamente pelo teste de Duncan a 5\% de probabilidade.

plantas, visto que neles, em geral, também aumentou a proporção de folhas infectadas, em relação às sadias. Esse fato pode ser atribuído à maior presença de fontes de inóculos na planta e/ou a diferenças de carga de frutos.

O citrumeleiro "Swingle” induziu área de projeção de copa (APC) semelhante à de outros portaenxertos vigorosos, indicando que neste porta-enxerto as copas tiveram grande desenvolvimento (Tabela 3). Apesar disso, a soma e a porcentagem de FoCC foram menores do que no limoeiro "Cravo", no "Volkameriana" e na tangerineira "Sunki". Isso possivelmente aconteceu porque o citrumeleiro "Swingle" proporcionou alta carga de frutos, decorrente de uma intensa florada na primavera. Nessa época, as temperaturas ainda são baixas, coincidindo com a ausência de ataque do minador dos citros (GREVE, 2004), fatores esses desfavoráveis ao CC. Nos meses seguintes, embora a temperatura aumentasse e ocorressem chuvas, a elevada carga de frutos deve ter impedido o acúmulo de reservas, reduzindo ou impedindo a emissão de brotações que seriam suscetíveis ao CC no final da primavera e no verão, que são as épocas mais propícias à doença (MONZANI, 2005).

As diferenças entre porta-enxertos, quanto ao número de frutos produzidos por planta (Nfr) em 2004, não foram significativas ao nível de 5\% (Tabela 4). Porém, em 2005, o porta-enxerto citrumeleiro "Swingle”, seguido dos limoeiros "Cravo" e "Volkameriano”, e do citrangeiro "Troyer” foram os que proporcionaram as maiores produções. Eles também revelaram os maiores índices de produtividade média, incluindo a tangerineira “Sunki”. Quanto à porcentagem média de frutos com lesões (\%FrCC), o limoeiro "Cravo", a tangerineira "Sunki" e o limoeiro "Volkameriana” apresentaram a maior \%FrCC, em relação aos porta-enxertos "Flying Dragon” e ao citrumeleiro "Swingle”. Verifica-se que, de certa forma, nos frutos, a incidência de CC esteve relacionada ao vigor conferido às plantas pelos porta-enxertos, o que pode ser justificado pela maior presença de inóculo nas folhas. A menor porcentagem de frutos com lesões de CC no "Flying Dragon” e no citrumeleiro "Swingle”, entretanto, também pode ser justificada pela menor presença de inóculo nas plantas, visto que, nesses porta-enxertos, o número de folhas com lesões foi menor (Tabela 1).

Em 2005, a \%FrCC foi inferior à de 2004 (Tabela 4). Isso pode ser atribuído às condições climáticas menos favoráveis à infecção da bactéria, visto que, nos meses de dezembro de 2004 e janeiro de 2005, período em que os frutos ainda são suscetíveis, as precipitações pluviométricas foram baixas, inferiores a $50 \mathrm{~mm}$.

O “Flying Dragon” mostra-se como o portaenxerto que induz a menor predisposição ao CC, sendo os seus frutos de boa qualidade. A indicação desse porta-enxerto como alternativa ao $\boldsymbol{P}$. trifoliata., no entanto, requer mais pesquisas porque nele o índice de produtividade foi inferior ao de plantas enxertadas sobre outros porta-enxertos (Tabela 4). Já as plantas enxertadas sobre o citrumeleiro "Swingle" apresentaram os maiores índices de produtividade e a menor incidência de CC, podendo ele ser indicado como uma alternativa ao uso do $\boldsymbol{P}$. trifoliata na Depressão Central do RS.

Os limoeiros “Cravo” e "Volkameriano”, a tangerineira "Sunki” e o citrangeiro "Troyer”, apesar de promoverem satisfatória produção de frutos, provocaram alta incidência de cancro cítrico, não sendo, portanto, aconselhável sua utilização em regiões com histórico ou risco dessa doença. 
Tabela 4 - Número de frutos produzidos (NFr), porcentagem de frutos com sintomas de cancro cítrico (\% FrCC) e índice de produtividade (IP) em laranjeiras-de-umbigo “Monte Parnaso” enxertadas sobre sete porta-enxertos 2004 e 2005. Butiá, RS, 2005

\begin{tabular}{|c|c|c|c|c|c|c|c|c|}
\hline Porta - enxertos & 2004 & 2005 & 2004 & 2005 & Média & 2004 & 2005 & Médias \\
\hline & \multicolumn{2}{|c|}{ NFr } & \multicolumn{3}{|c|}{$\%$ FrCC } & \multicolumn{3}{|c|}{$\mathrm{IP}\left(\mathrm{Kg} / \mathrm{m}^{2}\right)$} \\
\hline Citr. "Swingle” & 50,3 a & 113,0 a & $4,3 \mathrm{~b}$ & $2,2 \mathrm{ab}$ & $3,25 \mathrm{~d}$ & $4,0 \mathrm{a}$ & 9,1 a & 6,55 a \\
\hline Tang. "Sunki” & 42,3 a & $62,8 \mathrm{~b}$ & 13,3 a & $4,7 \mathrm{ab}$ & 9,00 & $4,0 \mathrm{a}$ & 5,7 & 4,85 \\
\hline "Volkameriana" & 41,3 a & 81,5 a & $8,5 \mathrm{ab}$ & $7,0 \mathrm{a}$ & 7,75 & $3,5 a b$ & $6,3 \mathrm{~b}$ & 4,90 \\
\hline Lim. “Cravo” & 37,5 a & $111,8 \mathrm{a}$ & 13,5 a & 7,8 a & 10,65 a & $3,3 a b$ & $8,2 \mathrm{ab}$ & $5,75 \mathrm{ab}$ \\
\hline "Flying Dragon" & 22,5 a & 7,8 с & $5,6 a b$ & $0,0 \mathrm{~b}$ & $2,78 \mathrm{~d}$ & $3,9 \mathrm{ab}$ & $1,4 \mathrm{c}$ & $2,65 \mathrm{c}$ \\
\hline Citr. "Troyer” & $27,0 \mathrm{a}$ & 110,3 a & $5,9 a b$ & $3,7 \mathrm{ab}$ & $4,80 \mathrm{c}$ & $2,3 \mathrm{~b}$ & $7,2 \mathrm{ab}$ & $4,75 \mathrm{~b}$ \\
\hline Lar. “Caipira” & 26,0 a & $72,25 \mathrm{~b}$ & $7,2 \mathrm{ab}$ & $1,5 \mathrm{ab}$ & $4,35 \mathrm{c}$ & $2,6 \mathrm{~b}$ & $5,4 \mathrm{~b}$ & $4,00 \mathrm{bc}$ \\
\hline $\mathrm{CV}$ & 20,10 & & & 38,51 & & 24,55 & 12,04 & 16,17 \\
\hline
\end{tabular}

Médias seguidas de mesma letra na coluna não difrem significativamente pelo teste de Duncam a 5\% de probabilidade.

\section{CONCLUSÕES}

O porta-enxerto citrumeleiro "Swingle", apesar de originar plantas vigorosas, proporciona elevado índice de produtividade e baixa incidência de cancro cítrico, podendo ser indicado como alternativa ao $\boldsymbol{P}$. trifoliata como porta-enxerto no Rio Grande do Sul.

O porta-enxerto "Flying Dragon”, induz nanismo e menor predisposição ao cancro cítrico, porém as plantas sobre ele enxertadas são as que apresentam o menor índice de produtividade.

Os limoeiros "Cravo" e "Volkameriana", apesar de proporcionarem altos índices de produtividade às plantas, somente devem ser indicados para uso como porta-enxerto em regiões sem histórico ou risco de cancro cítrico, porque ele induz grande predisposição das plantas a essa doença.

\section{AGRADECIMENTO}

Os autores agradecem o apoio do Conselho Nacional de Desenvolvimento Científico e Tecnológico (CNPq), que possibilitou a realização deste trabalho.

\section{REFERÊNCIAS}

AGOSTINI, J.P. et al. Relationship between development of citrus canker and rootstock for young "Valencia"orange trees in Missiones, Argentina. Proceedings of the Florida State Horticultural Society, Lake Alfred, v.98, p.19-22, 1985.

AMARAL, A.M. Cancro cítrico: permanente preocupação da citricultura no Brasil e no mundo. Brasília: Embrapa, 2003. 5p. (Comunicado técnico 86).

CANTEROS, B.I. Citrus canker in Argentina - Control, eradication, and current management. In: INTERNATIONAL CITRUS CANKER RESEARCH WORKSHOP, 2000, Ft. Pierce FL. Proceedings... Ft. Pierce: USDA, Agricultural Research Service and U.S. Horticultural Research Laboratory, 2001. p.20-22.
CHRISTIANO, R.S.C. Componentes monocíclicos do cancro cítrico no sistema Xanthomonas axonopodis pv. citri - Limão Tahiti - Plyllocnistis citrella, sob condições controladas. 2003. 104f. Dissertação (Mestrado em Fitopatologia) - Programa de Pós-graduação em Fitopatologia, Escola Superior de Agricultura "Luiz de Queiroz”. Universidade de São Paulo, Piracicaba.

DANOS, E. et al. Progress of citrus canker in some species and combinations in Argentina. Proceedings of the Florida State Horticultural Society, Lake Alfred, v.94, p.15-18, 1981.

DANOS, E. et al. Temporal and spatial spread of citrus canker within groves. Phytopathology, Saint Pierre, v.74, p.904908, 1984

GREVE, C. Aspectos bioecológicos das fases imaturas de Plyllocnistis citrella Stainton (Lepidoptera: Gracillariidae), em pomares de citrus sinensis Var. Valência sob dois sistemas de cultivo. 2004. 107f. Dissertação (Mestrado em Biologia Animal) - Programa de Pós-graduação em Biologia Animal, Instituto de Biociências, Universidade Federal do Rio Grande do Sul, Porto Alegre.

LEITE JÚNIOR, R.P.; SANTOS, S.D. Suscetibilidade do limão Siciliano (Citrus lemon), enxertados sobre diferentes portaenxertos, ao cancro cítrico causado por Xanthomonas campestris pv. citri. Fitopatologia Brasileira, Brasília, v.13, p.353-358, 1988.

LEITE JÚNIOR, R.P. Cancro cítrico: prevenção e controle no Paraná. Londrina: IAPAR, 1990. 51p. (Circular Técnica, 61).

MARTINS, F.T. Comportamento de laranjeira "Valência" e tangerineira "Montenegrina" propagadas por estaquia e enxertia. 2005. 66f. Dissertação (Mestrado em FitotecniaHorticultura) - Programa de Pós-graduação em Fitotecnia, Faculdade de Agronomia, Universidade Federal do Rio Grande do Sul, Porto Alegre.

MONZANI, R.M. Incidência e severidade de cancro cítrico em laranjeiras “Monte Parnaso" com ensacamento, poda sanitária, desbrote e tratamento cúprico. 2005. $88 \mathrm{f}$. Dissertação (Mestrado em Fitotecnia-Fitossanidade) - Programa de Pós-graduação em Fitotecnia, Faculdade de Agronomia, Universidade Federal do Rio Grande do Sul, Porto Alegre. 
NAMEKATA, T. et al. Comportamento de uma coleção de citros submetida à contaminação ao cancro cítrico, causado pela bactéria Xanthomonas campestris pv. citri. Laranja, Cordeirópolis, v.13, n.2, p.757-775, 1992.

OLIVEIRA, R.P. et al. Manual técnico sobre o cancro cítrico. Pelotas. Embrapa Clima Temperado, 2001. 24p. (Embrapa Clima Temperado, Circular Técnico, 27).

RODRIGUES NETO, J.; RIBEIRO, J.G.B. Manual técnico de procedimentos do cancro cítrico. Brasília: MAPA/DAS/ DDIV, 2002. 66p.

ROSETTI, V.V. Manual ilustrado de doenças dos citros. Piracicaba: Fealq - Fundecitrus, 2001. 207p.

SCHÄFER, G. et al. Produção e desenvolvimento da tangerineira "Montenegrina” propagada por enxeretia e estaquia, no Rio Grande do Sul. Revista Brasileira de Fruticultura, Jaboticabal-SP, v.23, n.3, p.668-672, 2001.
SCHUBERT, T.S.; SUN, X. Bacterial citrus canker. Florida: Departament of Agriculture \& Conservancy Services, 2003. 6p. (Plant Pathology Circular, 377).

THEISEN, S. Incidência de cancro cítrico (Xanthomonas citri pv citri) em pomar de laranjeiras "Valência” sob concentrações e freqüências de pulverizações cúpricas. 2004. 63f. Dissertação (Mestrado em Fitotecnia-Horticultura) Programa de Pós-graduação em Fitotecnia, Faculdade de Agronomia, Universidade Federal do Rio Grande do Sul, Porto Alegre.

VERNIERE, C.J. et al. Disease development and symptom expression of Xanthomonas axonopodis pv. citri in various citrus plant tissues. Phytopathology, St Paul, v.93, n.7, p.832843, 2003.

WUTSCHER, H.K. Citrus roostocks. Horticultural Reviews, New York, v.1, p.237-269, 1979. 